Ophthalmologe 2019 116 (Suppl 1):S1-S9 https://doi.org/10.1007/s00347-018-0810-1 Published online: 7 November 2018 (c) Springer Medizin Verlag GmbH, ein Teil von Springer Nature 2018

CrossMark
Professional Association of German Ophthalmologists (Berufsverband der Augenärzte Deutschlands e. V., BVA) ${ }^{1} \cdot$ German Ophthalmological Society (Deutsche Ophthalmologische Gesellschaft, DOG) ${ }^{2}$ - German Retina Society e.V. (Retinologische Gesellschaft e. V., RG) ${ }^{3}$

'Professional Association of German Ophthalmologists (Berufsverband der Augenärzte Deutschlands e.V.), Düsseldorf, Germany

${ }^{2}$ German Society of Ophthalmology (Deutsche Ophthalmologische Gesellschaft), Munich, Germany

${ }^{3}$ German Retina Society (Retinologische Gesellschaft e. V.), Freiburg, Germany

\section{Statement of the BVA, the DOG, and the RG on treatment of choroidal neovascularization in diseases other than neovascular age-related macular degeneration}

\section{Dated Ocotber 2017}

\section{Key messages}

1. Choroidal neovascularizations $(\mathrm{CNV})$ occur not only in age-related macular degeneration (AMD), but also in numerous other macular and retinal disorders of varying etiology and, if left untreated, can cause irreversible visual loss.

2. The diagnosis of CNV as well as the indication for treatment should be made in the same way as in neovascular AMD:

- On initial diagnosis: best-corrected visual acuity, fundus examination, optical coherence tomography (OCT), and fluorescein angiography

- At follow-up: best-correct visual acuity, fundus examination, OCT, and, depending on findings, fluorescein angiography

3. Active CNV should be treated with intravitreal operative medication

The German version of this article can be found under https://doi.org/10.1007/s00347018-0679-z.
(IVOM) using vascular endothelial growth factor (VEGF) inhibitors if patients have visual acuity of at least 0.05 or if there is sufficient reason to assume that visual acuity could increase to over 0.05 under treatment.

4. Underlying disorders can include, e. g., (only a selection is presented here):

- High myopia

- Angioid streaks

- Central serous chorioretinopathy

- Active and inactive uveitis of varying etiology, including retinochoroiditis, chorioretinitis, and choroiditis

- Eye injuries

- Retinal dystrophies, e.g., best disease and pattern dystrophies

- Idiopathic CNV

- Subretinal masses (osteomas, hamartomas, nevi)

If $\mathrm{CNV}$ is not present as a complication in the above-mentioned disorders, IVOM with VEGF inhibitors should not be performed.
5. Ranibizumab and aflibercept are approved in Germany for the treatment of CNV secondary to pathologic myopia.

6. Ranibizumab has been approved in Germany since 12/2016 for the treatment of $\mathrm{CNV}$ in disorders other than neovascular AMD and pathologic myopia irrespective of the underlying disease. The other VEGF inhibitors, aflibercept and bevacizumab, can be used off-label.

7. Due to its overall significantly namic therapy (PDT) should only be used in exceptional cases and extrafoveal localization.

8. After one initial intravitreal administration of VEGF inhibitors, further CNV activity should be monitored monthly for the first 6 months (see point 2). In the case of persisting or recurrent activity, repeated IVOM should be performed. Depending on disease course, the follow-up interval might be extended 6 months after the last IVOM. In individual justified cases poorer treatment results, photody- 
(e.g., patients requiring frequent re-injections), a different treatment regimen (e.g., treat and extend) can be considered in the further course.

9. If visual acuity drops below 0.05 on anti-VEGF treatment, or no further positive treatment outcome is expected (e.g., in the presence of atrophy and/or fibrosis), treatment should be discontinued, unless there is a clear possibility that visual acuity could increase again to over 0.05 under treatment.

10. If no improvement is seen under therapy with a certain VEGF inhibitor, or if deterioration occurs, one can consider switching to an alternative VEGF inhibitor.

\section{Introduction}

In addition to age-related macular degeneration (AMD), there are numerous other retinal diseases of varying etiology that can be associated with choroidal neovascularizations $(\mathrm{CNV})$. As in AMD, severe irreversible visual loss can occur if left untreated.

The following article discusses examples of the more common underlying diseases.

\subsection{High myopia}

High myopia is generally defined as a refractive error of $\geq-6$ diopters spherical equivalent or an axial length $\geq 26.5 \mathrm{~mm}$. A distinction must be made from pathologic myopia, which is additionally characterized by typical fundus lesions, e.g., myopic cracks, chorioretinal atrophy, and pigment changes [1].

Population-based studies estimate the incidence of high myopia in Europe to be approximately $2.7 \%$, with increasing incidence and prevalence at a younger age $[2,3]$. A dependence on ethnicity is apparent, with a higher prevalence among Asians compared with Caucasians [4]. Other factors that appear to be associated with myopia include urban environment and higher education levels [5].

There are currently no reliable figures on the frequency of pathologic myopia in Germany. Up to $3.2 \%$ of Asian populations exhibit myopia-induced pathological fundus changes [6]. It is assumed that approximately $5-10 \%$ of patients with pathologic myopia develop CNV [1]. From this, one can estimate an overall prevalence of approximately $0.2 \%$ in the total population for Europe/ Germany. The natural disease course is often unfavorable, involving progressive loss of visual acuity, particularly in the case of subfoveal CNV [7]. Compared with AMD, the affected group is younger and often still in working age, meaning that, if left untreated, considerable socioeconomic effects are associated with this complication. Overall, CNV secondary to myopia is considered the commonest cause of vision loss in young people aged $\leq 50$ years [1].

\subsection{Angioid streaks}

Angioid streaks (AS) is a descriptive term for reddish-brown lines visible on fundoscopy, which are typically circular around the optic disc, from where they radiate into the periphery [8]. The term originates from their similarity to vessels on fundoscopy. Histopathologically, AS are small breaks in Bruch's membrane. There are numerous underlying systemic diseases that can be associated with AS. With an estimated prevalence of 1:25,000-100,000, pseudoxanthoma elasticum (PXE) is the most frequent of these diseases [9]. However, AS also occurs in other disorders, such as PXElike syndromes, e.g., generalized arterial calcification of infancy (GACI), various hemoglobinopathies, e.g., $\beta$-thalassemia and sickle cell anemia, as well as Paget's disease in rare cases [8]. As long as the retinal pigment epithelium and the photoreceptors are intact, AS generally have no serious effects on patients' vision. However, they predispose to the development of CNV, which manifests in the majority of patients in the disease course [10]. CNV secondary to AS are typically classic membranes (type 2). If left untreated, these CNV often have a poor prognosis due to their aggressiveness and foveal involvement and generally result in significant, irreversible loss of visual acuity [8]. Since AS-related diseases are usually systemic diseases, the interdisciplinary treatment of patients, e.g., in collaboration with internists, plays an important role. However, it has been shown that vision impairment, at least with regard to PXE patients, had the greatest impact on quality of life compared with other comorbidities [11]. For further information, the reader is referred to the statement from the DOG, RG, and BVA on the treatment of choroidal neovascularization in PXE.

\subsection{Central serous chorioretinopathy}

Central serous chorioretinopathy (CSC) is a relatively common disease of the ocular fundus, which results in localized serous detachment of the neurosensory retina in the macular region, often accompanied by focal detachment of the retinal pigment epithelium [12]. Another characteristic feature frequently seen is an increased choroidal thickness, indicating that the choroid may play an important pathogenetic role [13]. The disease typically affects younger male patients [14]. A link to increased stress levels [12], as well as the systemic use of glucocorticoids, has been described [15]. Due to the positive effects sometimes observed for aldosterone receptor antagonists, mineralocorticoid receptor involvement is also discussed [16].

In general, the acute form of CSC is a self-limiting disease that improves spontaneouslywithin around 3-4 months [17]. It is important to distinguish this form from chronic CSC, in which persistent angiographic leakage in the macular region, chronic subretinal fluid, pigment changes, and atrophic lesions can still be found even after 6 months. The chronic form in particular is associated with the development of $\mathrm{CNV}$, which can lead to irreversible loss of vision. The incidence of CNV secondary to chronic CSC is put at $4-8 \%$ [18]. The recommendations below relate exclusively to the treatment of CSC-related CNV. Further information on CSC and its treatment can be found in a separate statement from the DOG, RG, and BVA. 


\subsection{Active and inactive uveitis of varying etiology, including retinochorioiditis, chorioretinitis, and chorioiditis}

In principle, a multitude of intraocular inflammatory disorders are associated with the development of CNV. It occurs particularly in punctate inner choroidopathy (PIC), multifocal choroiditis, presumed ocular histoplasmosis syndrome (POHS), Vogt-Koyanagi-Harada syndrome, toxoplasmosis, and serpiginous choroiditis [19]. A study on 648 patients determined the frequency of $\mathrm{CNV}$ to be approximately $2 \%$, irrespective of the underlying etiology [20]. If left untreated, the formation of $\mathrm{CNV}$ is associated with a poor prognosis due to the often young age of patients and the aggressive course of the disease [21-23]. The diagnosis of $\mathrm{CNV}$ secondary to uveitis can be hampered, for example, by concomitant inflammatory macular edema. The treatment recommendations below are explicitly intended only for cases with proven CNV. With regard to the treatment of inflammatory macular edema secondary to uveitis, the reader is referred to the relevant statement from the DOG, RG, and BVA. Furthermore, when establishing the diagnosis of $\mathrm{CNV}$, a comprehensive diagnostic workup (if this has not already taken place) should be carried out to identify possible underlying diseases and, if applicable, a treatment of the underlying disease should be initiated.

\subsection{Eye injuries}

The development of CNV following traumatic eye injury, while rare, is potentially vision threatening. Patients in whom the choroid has ruptured in the perifoveal region, generally resulting from blunt occur trauma, are at greatest risk. The risk of developing CNV following choroidal rupture is put at approximately 5\% [24]. In rare cases, lasers can also cause traumatic or iatrogenic chorioretinal injury. Pathogenetically, disruption in the region of the retinal pigment epithelium and Bruch's membrane as a result of intense laser energy likely plays a crucial role $[25,26]$.

\subsection{Retinal dystrophies}

There are a number of retinal dystrophies that, in rare cases, are associated with CNV. However, due to their rarity, no large relevant case series are available. By way of example, attention should be drawn to frequent associations with Best disease, adult vitelliform macular dystrophy, and pattern dystrophies [27-30]. In addition, CNV is often also seen in Sorsby fundus dystrophy and, if so, is associated with a relatively aggressive course [31].

\subsection{Idiopathic CNV}

Idiopathic $\mathrm{CNV}$ is defined as $\mathrm{CNV}$ in patients younger than 50 years once possible underlying ocular or systemic diseases have been excluded [32]. There are no reliable figures on the frequency of idiopathic CNV; however, it is a rare condition. Although the natural course of idiopathic CNV is often relatively mild compared with other entities, it is subject to considerable variability given, e.g., its sometimes extrafoveal localization [32].

\subsection{Subretinal tumors}

In principle, all subretinal masses can cause CNV. Hereof, CNV secondary to osteomas, sclerochoroidal calcifications, and nevi have most frequently been described. Osteomas in particular are most likely to cause structural changes in the photoreceptor-pigment epithelium complex predisposing to subsequent $\mathrm{CNV}$ development. Although masses as such rarely cause a reduction in visual acuity, $\mathrm{CNV}$ is often associated with a massive loss in visual acuity [33].

\section{Treatment methods/study results}

\subsection{Anti-VEGF therapy}

\subsubsection{The RADIANCE study}

The RADIANCE study [34] investigated the efficacy and safety of $0.5 \mathrm{mg}$ intravitreal ranibizumab in patients with myopic CNV compared to PDT over 12 months in a randomized, double-blind, multicenter, controlled phase III study. A total of 277 patients were included and randomized to three arms:

1. Ranibizumab $0.5 \mathrm{mg}$, treatment regimen according to "stability criteria," defined as no change in best-corrected visual acuity (BCVA) compared with the two previous monthly controls.

2. Ranibizumab $0.5 \mathrm{mg}$, treatment regimen according to "disease activity criteria," defined as visual impairment due to intra- or subretinal fluid or active leakage secondary to a $\mathrm{CNV}$ lesion determined using OCT and/or fluorescein angiography.

3. PDT; treatment with ranibizumab was possible from the third month.

The primary endpoint was BCVA at 3 months.

- Ranibizumab therapy was superior to PDT at 3 months in terms of BCVA (group 1: +10.5 letters, group 2: +10.6 letters, group 3: +2.2 letters).

- The improvement in vision was accompanied by a reduction in central retinal thickness.

- Further improvement in visual acuity was observed at 12 months in the groups treated primarily with ranibizumab (group 1: +13.8 letters, group 2: +14.4 letters).

- Although patients in group 3, that were able to receive additional treatment with $0.5 \mathrm{mg}$ intravitreal ranibizumab from month 3 , subsequently showed an increase in visual acuity, they failed to reach the level of patients treated primarily with ranibizumab (+9.3 letters) at 12 months.

- Treatment monitoring by means of disease activity criteria (group 2) was not inferior to visual acuitymonitored treatment.

- Patients received on average 4.6 injections in group 1 and 3.5 injections in group 2 over a 12-month period. In all, $50.9 \%$ of patients needed $1-2$ injections, $34.5 \% 3-5$, and $14.7 \%$ 6-12 injections.

- The ocular and non-ocular safety profile was in line with ranibizumab in other indications. 


\subsubsection{The MYRROR study}

The MYRROR study [35] investigated the efficacy and safety of $2 \mathrm{mg}$ intravitreal aflibercept in patients with myopic CNV in a multicenter, randomized, doubleblind, controlled phase III study. A total of 122 patients were included and randomized to two arms:

1. Aflibercept $2 \mathrm{mg}$, re-injections according to activity criteria at monthly follow-up (visual acuity, retinal thickness, intra-/subretinal fluid, CNV activity, bleeding, at examiner's discretion); if criteria were negative, a sham injection was performed.

2. Monthly fixed sham injection. From week 24 , treatment with $2 \mathrm{mg}$ aflibercept as in group 1 .

The primary endpoint was BCVA at 24 weeks.

- At 24 weeks, treatment with aflibercept proved superior to sham injections in terms of BCVA (group 1: +12.1 letters, group 2: -2 letters).

- At 48 weeks, a further improvement in visual acuity (+13.5 letters) was seen in the patient group treated primarily with aflibercept (group 1).

- Although patients in the group that were able to receive treatment with aflibercept from week 24 (group 2) also showed an increase in visual acuity (+3.9 letters) at 48 weeks, they did not reach the level of patients treated primarily with aflibercept.

- The improvement in vision was accompanied by a reduction in central retinal thickness.

- On average, 4.2 injections were administered in group 1 and 3.0 injections in group 2 over a 12 -month period.

- The ocular and non-ocular safety profile was in line with aflibercept treatment in other indications.

\subsubsection{The MINERVA study}

The MINERVA study [36] investigated the efficacy and safety of $0.5 \mathrm{mg}$ intravitreal ranibizumab in patients with $\mathrm{CNV}$ due to diseases other than AMD and myopia. The study was a multicenter, double-blind, randomized, controlled phase III study. A total of 178 patients with a variety of underlying diseases were included. Patients were randomized to two groups:

1. Ranibizumab $2 \mathrm{mg}$, re-injections according to activity criteria at monthly follow-up (visual acuity, retinal thickness, intra-/subretinal fluid, CNV activity, bleeding, at examiner's discretion); if criteria were negative, a sham injection was administered.

2. Monthly fixed sham injection. From month 2, treatment with $0.5 \mathrm{mg}$ ranibizumab as in group 1.

The primary endpoint was BCVA at 2 months.

- At 2 months, the ranibizumabtreated group showed significantly better results in terms of BCVA compared with the sham treatment group (group 1: 9.5 letters, group 2:-0.4 letters).

- Once it became possible to use ranibizumab in group 2 from month 2 , an increase in visual acuity (+9.3 letters) was seen at 12 months, which reached the level of patients treated primarily with ranibizumab (group 1: +11 letters).

- The improvement in vision was accompanied by a reduction in central retinal thickness.

- The two groups received a comparable number of injections (group 1: 5.8, group 2: 5.4).

- The ocular and non-ocular safety profile was in line with ranibizumab treatment in other indications.

\subsubsection{Other studies}

In addition to these three approval trials, numerous other studies on the treatment of $\mathrm{CNV}$ in diseases other than AMD have been conducted using various VEGF inhibitors. However, these are mostly retrospective case series or case reports with low evidence levels. Therefore, the following are only a few examples of larger studies that have yielded additional information. For further information, the reader is referred to the statements of the DOG, RG, and BVA on the treatment of myopic CNV and CNV in pseudoxanthoma elasticum.

With regard to myopic $\mathrm{CNV}$, and in addition to the studies on ranibizumab and aflibercept mentioned above, smaller randomized controlled trials have also been published that compared the treatment of CNV with bevacizumab and PDT [37] and PDT and laser coagulation [38]. Treatment with bevacizumab proved to be superior to the other treatments. Based on a large meta-analysis, the efficacy of bevacizumab appears to be comparable to that of ranibizumab [39].

There are numerous case series, some of which are prospective but most of which are retrospective, on bevacizumab for other indications. Here again, good efficacy that appears similar to that of ranibizumab was generally seen, e.g., in angioid streaks [8], CSC-related CNV [40, 41], inflammatory CNV [19], and idiopathic CNV [40, 42]. With regard to aflibercept, there are at present primarily isolated case reports that similarly point to good efficacy [41, 43, 44]. An open-label phase I/II study has also been published on the treatment of CNV secondary to POHS, demonstrating the efficacy of aflibercept treatment in POHSrelated $\mathrm{CNV}$ with two different treatment strategies [45].

\subsection{Photodynamic therapy}

Based on the results of the VIP study, verteporfin is approved in Germany for the treatment of subfoveal $\mathrm{CNV}$ in myopia [46]. Although the study showed that PDT is able to stabilize visual acuity, improvements in vision were rarely observed. Based on the results of the RADIANCE study (see above), PDT therapy was clearly inferior to ranibizumab. Therefore, PDT is no longer recommended as a firstline treatment in myopic CNV. PDT is currently not approved for the treatment of $\mathrm{CNV}$ in diseases other than AMD and myopia. However, based on individual case series, a stabilization of vision also appears to be possible here [8, 42, 47-50]. PDT can be considered if $\mathrm{CNV}$ is in a clear extrafoveal location. However, improvements in visual acuity in line with those reported for VEGF inhibitors were rarely observed [51]. Good functional results were seen for CSC-related CNV in particular [52, 53]. Combining PDT with a VEGF inhibitor 
could potentially also be beneficial in this setting [54]. However, there are no large studies in this regard to date. Therefore, on the whole, PDT should also not be recommended as a first-line treatment for these indications.

\section{Treatment recommendations}

\subsection{Diagnosis and indication}

In addition to visual acuity testing (BCVA with undilated pupils and under standardized conditions) and fundus examination in mydriasis, the fluorescein angiograph (FAG) forms the central basis for the detection and documentation of $\mathrm{CNV}$ in retinal diseases other than AMD. Therefore, an FAG must be carried out (except in the case of known fluorescein allergy) prior to all initial treatments in order to document the treatment indication (see brief statement from the DOG, RG, and BVA on performing and indicating IVOM). In addition, spectraldomain (SD)-OCT should also be performed prior to all initial treatments and is not only of great value for the initial diagnosis but also as an important parameter for treatment monitoring during follow-up examinations.

In addition to the diagnosis of CNV, the precise differential diagnosis of underlying diseases is of particular importance in this patient group. This is particularly relevant since, in many cases, interdisciplinary care and treatment of the underlying disease is required.

Follow-up examinations should include BCVA, a fundus examination in mydriasis, and SD-OCT. In unclear cases, a repeated FAG is also recommended.

Follow-up examinations should take place monthly after the last IVOM for a period of at least 6 months. Thereafter, "scarring" of the CNV can be clinically assumed and follow-up intervals extended where appropriate depending on findings.

\subsection{Treatment approaches}

Ranibizumab and aflibercept (see above) as well as PDT (subfoveal location of the $\mathrm{CNV}$ ) are currently approved in Germany for the treatment of CNV in myopia on the basis of relevant approval studies. However, due to significantly better results on comparison, treatment with ranibizumab or aflibercept is essentially preferred over PDT.

Ranibizumab has been approved in Germany since 12/2016 for the treatment of $\mathrm{CNV}$ in the setting of disorders other than neovascular AMD and pathological myopia irrespective of the underlying disease. The other VEGF inhibitors, aflibercept and bevacizumab, can be used off-label. When doing so, the basic requirements for the use of off-label drugs described in previous statements by the BVA, DOG, and RG must be fulfilled.

Due to the often highly varying individual responses to VEGF inhibitor therapy and possible disease inactivity following only one initial treatment, one should start with a one IVOM. Further lesion activity is then monitored on the basis of subsequent monthly follow-ups. In the case of persistent or renewed morphologically visible lesion activity, repeated IVOM is performed in each instance.

More than 6 months following the last IVOM, follow-up examinations can be carried out at longer intervals where appropriate. In justified individual cases (e.g., patients requiring frequent re-injections), a different treatment regimen (e.g., treat and extend) can be considered in the further course.

If $\mathrm{CNV}$ is concomitant to angioid streaks, the particular aggressiveness of the disease and the need for more IVOM needs to be taken into account. Therefore, if CNV occurs, persists, or recurs in angioid streaks, a series of three IVOMs may be indicated.

The criteria for repeat IVOM are based on the recommendations for the treatment of neovascular AMD:

1. Subretinal fluid (OCT)

2. Persistence or increase in diffuse retinal thickening

3. Increased intraretinal cystoid fluid (OCT)

4. Increased pigment epithelial detachment (OCT)

5. New sub- or intraretinal bleeding (fundoscopy)

6. De novo reduction in visual acuity due to $\mathrm{CNV}$
In unclear cases, a FAG can also be performed, with the detection of active CNV serving as a criterion for re-injection.

If patients fail to respond or further deterioration is seen during ongoing therapy with one particular VEGF inhibitor, one can consider switching to another VEGF inhibitor or, in some cases, performing a PDT. However, it should be borne in mind in such cases that this may constitute off-label use.

If visual acuity falls below 0.05 under therapy, or if morphological findings (e.g., atrophy and/or fibrosis) indicate no further positive therapeutic impact, treatment should be discontinued. In individual cases, treatment may also be indicated in visual acuity of under 0.05 , assuming there is sufficient evidence to suggest that vision could increase again to 0.05 under treatment.

\subsection{Performing treatment}

The reader is referred here to the statement "Die Anti-VEGF-Therapie bei der neovaskulären altersabhängigen Makuladegeneration: Therapeutische Strategien" ("Anti-VEGF therapy in neovascular age-related macular degeneration: treatment Strategies"), dated November 2014, and the "Empfehlung der Deutschen Ophthalmologischen Gesellschaft, der Retinologischen Gesellschaft und des Berufsverbandes der Augenärzte Deutschlands für die Durchführung von intravitrealen Injektionen (IVI)" ("Recommendation of the German Ophthalmological Society, the German Retina Society, and the German Professional Association of Ophthalmologists on the performance of Intravitreal Injections (IVI)").

\section{Corresponding address}

German Ophthalmological Society (Deutsche Ophthalmologische Gesellschaft, DOG)

German Society of Ophthalmology (Deutsche Ophthalmologische Gesellschaft) Platenstr. 1, 80336 Munich, Germany geschaeftsstelle@dog.org

Editorial board.. Priv.-Doz. Dr. Martin Gliem, University Eye Hospital Bonn, Bonn, Germany (head of board) 
Prof. Dr. Bernd Bertram, Ophthalmology Practice, Aachen, Germany

Prof. Dr. Norbert Bornfeld, University Eye Hospital Essen, Essen, Germany

Prof. Dr. Frank G. Holz, University Eye Hospital Bonn, Bonn, Germany

Prof. Dr. Mathias Maier, University Eye Hospital, TU Munich, Germany

Prof. Dr. Daniel Pauleikhoff, Ophthalmology Department at the St. Franziskus Hospital Münster, Germany

\section{Compliance with ethical guidelines}

Conflict of interests. See $\bullet$ Table 1 in the Appendix.

This article does not contain any studies with human participants or animals performed by any of the authors.

The supplement containing this article is not sponsored by industry. 


\section{Appendix}






\section{References}

1. Neelam K, Cheung CM, Ohno-Matsui K, Lai TY, Wong TY (2012) Choroidal neovascularization in pathological myopia. Prog Retin Eye Res 31:495-525

2. Williams KM, Verhoeven VJ, Cumberland $P$ et al (2015) Prevalence of refractive error in Europe: the European Eye Epidemiology ( $E(3))$ Consortium. Eur JEpidemiol 30:305-315

3. Mirshahi A, Ponto KA, Laubert-Reh D et al (2016) Myopia and cognitive performance: results from the Gutenberg Health Study. Invest Ophthalmol Vis Sci 57:5230-5236

4. Wolfram C, Hohn R, Kottler U et al (2014) Prevalence of refractive errors in the European adult population: the Gutenberg Health Study (GHS). Br J Ophthalmol 98:857-861

5. Xu L, Li J, Cui T et al (2005) Refractive error in urban and rural adult Chinese in Beijing. Ophthalmology 112:1676-1683

6. Liu HH, Xu L, Wang YX, Wang S, You QS, Jonas JB (2010) Prevalence and progression of myopic retinopathy in Chinese adults: the Beijing Eye Study. Ophthalmology 117:1763-1768

7. Miller DG, Singerman LJ (2001) Natural history of choroidal neovascularization in high myopia. Curr Opin Ophthalmol 12:222-224

8. Gliem M, Finger RP, Fimmers R, Brinkmann $\mathrm{CK}_{,}$ Holz FG, Charbel Issa P (2013) Treatment of choroidal neovascularization due to angioid streaks: a comprehensive review. Retina 33:1300-1314

9. Gliem M, Zaeytijd JD, Finger RP, Holz FG, Leroy BP, Charbel Issa P (2013) An update on the ocular phenotype in patients with pseudoxanthoma elasticum. Front Genet 4:14

10. Gliem M, Muller PL, Birtel J, Hendig D, Holz FG, Charbel Issa P (2016) Frequency, phenotypic characteristics and progression of atrophy associated with a diseased Bruch's membrane in pseudoxanthoma elasticum. Invest Ophthalmol Vis Sci 57:3323-3330

11. Finger RP, Fenwick E, Marella $M$ et al (2011) The relative impact of vision impairment and cardiovascular disease on quality of life: the example of pseudoxanthoma elasticum. Health Qual Life Outcomes 9:113

12. Daruich A, Matet A, Dirani A et al (2015) Central serous chorioretinopathy: recent findings and new physiopathology hypothesis. Prog Retin Eye Res 48:82-118

13. Mrejen S, Spaide RF (2013) Optical coherence tomography: imaging of the choroid and beyond. Surv Ophthalmol 58:387-429

14. Spaide RF, Campeas L, Haas A et al (1996) Central serous chorioretinopathy in younger and older adults. Ophthalmology 103:2070-2079 (discussion 2079-80)

15. Bouzas EA, Karadimas P, Pournaras CJ (2002) Central serous chorioretinopathy and glucocorticoids. Surv Ophthalmol 47:431-448

16. Bousquet E, Beydoun T, Rothschild PR et al (2015) Spironolactone for nonresolving central serous chorioretinopathy: a randomized controlled crossover study. Retina 35:2505-2515

17. Yannuzzi LA (2010) Central serous chorioretinopathy: a personal perspective. Am J Ophthalmol 149:361-363

18. Peiretti E, Ferrara DC, Caminiti G, Mura M, Hughes J (2015) Choroidal neovascularization in caucasian patients with longstanding centra serous chorioretinopathy. Retina 35:1360-1367
19. Mansour AM, Arevalo JF, Ziemssen $F$ et al (2009) Long-term visual outcomes of intravitreal bevacizumab in inflammatory ocular neovascularization. Am JOphthalmol 148(e2):310-316

20. Perentes $Y$, Van Tran T, Sickenberg M, Herbort CP (2005) Subretinal neovascular membranes complicating uveitis: frequency, treatments, and visual outcome. Ocul Immunol Inflamm 13:219-224

21. Kleiner RC, Ratner CM, Enger C, Fine SL (1988) Subfoveal neovascularization in the ocular histoplasmosis syndrome. A natural history study. Retina 8:225-229

22. Brown J Jr., Folk JC, Reddy CV, Kimura AE (1996) Visual prognosis of multifocal choroiditis, punctate inner choroidopathy, and the diffuse subretinal fibrosis syndrome. Ophthalmology 103:1100-1105

23. Yang P, Ren Y, Li B, Fang W, Meng Q, Kijlstra A (2007) Clinical characteristics of Vogt-Koyanagi-Harada syndrome in Chinese patients. Ophthalmology 114:606-614

24. Wood CM, Richardson J (1990) Chorioretinal neovascular membranes complicating contusional eye injuries with indirect choroidal ruptures. $\mathrm{Br} J$ Ophthalmol 74:93-96

25. Chen CJ, Urban LL, Nelson NC, Fratkin JD (1998) Surgical removal of subfoveal iatrogenic choroidal neovascular membranes. Ophthalmology 105:1606-1611

26. Chan WM, Li KK, Liu DT, Lai TY, Chong KK, Lam DS (2003) Photodynamic therapy with verteporfin in laser-induced choroidal neovascularization. Am J Ophthalmol 136:565-567

27. Marano F, Deutman AF, Leys A, Aandekerk AL (2000) Hereditary retinal dystrophies and choroidal neovascularization. Graefes Arch Clin Exp Ophthalmol 238:760-764

28. Sodi A, Murro V, Caporossi O et al (2015) Long-term results of photodynamic therapy for choroidal neovascularization in pediatric patients with best vitelliform macular dystrophy. Ophthalmic Genet 36:168-174

29. Parodi MB, lacono $P$, Cascavilla $M$, Zucchiatti I, Kontadakis DS, Bandello F (2010) Intravitreal bevacizumab for subfoveal choroidal neovascularization associated with pattern dystrophy. Invest Ophthalmol Vis Sci 51:4358-4361

30. Andrade RE, Farah ME, Costa RA (2003) Photodynamic therapy with verteporfin for subfoveal choroidal neovascularization in best disease. Am J Ophthalmol 136:1179-1181

31. Gliem M, Muller PL, Mangold E et al (2015) Sorsby fundus dystrophy: novel mutations, novel phenotypic characteristics, and treatment outcomes. Invest Ophthalmol Vis Sci 56:2664-2676

32. Ho AC, Yannuzzi LA, Pisicano K, DeRosa J (1995) The natural history of idiopathic subfoveal choroidal neovascularization. Ophthalmology 102:782-789

33. Papastefanou VP, Pefkianaki M, Al Harby L et al (2016) Intravitreal bevacizumab monotherapy for choroidal neovascularisation secondary to choroidal osteoma. Eye (Lond) 30:843-849

34. Wolf S, Balciuniene VJ, Laganovska $G$ et al (2014) RADIANCE: a randomized controlled study of ranibizumab in patients with choroidal neovascularization secondary to pathologic myopia. Ophthalmology 121:682-692.e2

35. Ikuno Y, Ohno-Matsui K, Wong TY et al (2015) Intravitreal aflibercept injection in patients with myopicchoroidal neovascularization: the MYRROR Study. Ophthalmology 122:1220-1227

36. Lai TYY, Staurenghi G, Lanzetta P et al (2017) Efficacy and safety of ranibizumab for the treatment of choroidal neovascularization due to uncommon cause: twelve-month results of the MINERVA study. Retina. https://doi.org/10.1097/ IAE.0000000000001744

37. Ruiz-Moreno JM, Arias L, Montero JA, Carneiro A, Silva R (2013) Intravitreal anti-VEGF therapy for choroidal neovascularisation secondary to pathological myopia: 4-year outcome. $\mathrm{Br} J$ Ophthalmol 97:1447-1450

38. Parodi MB, lacono P, Papayannis A, Sheth $S$, Bandello F (2010) Laser photocoagulation, photodynamic therapy, and intravitreal bevacizumab for the treatment of juxtafoveal choroidal neovascularization secondary to pathologic myopia. Arch Ophthalmol 128:437-442

39. Zhu Y, Zhang T, Xu G, Peng L (2016) Antivascular endothelial growth factor for choroidal neovascularisation in people with pathological myopia. Cochrane Database Syst Rev. https://doi. org/10.1002/14651858.CD011160.pub2

40. Chan WM, Lai TY, Liu DT, Lam DS (2007) Intravitreal bevacizumab (avastin) for choroidal neovascularization secondary to central serous chorioretinopathy, secondary to punctate inner choroidopathy, or of idiopathic origin. Am J Ophthalmol 143:977-983

41. Chhablani J, Kozak I, Pichi F et al (2015) Outcomes of treatment of choroidal neovascularization associated with central serous chorioretinopathy with intravitreal antiangiogenic agents. Retina 35:2489-2497

42. Kang HM, Koh HJ (2013) Intravitreal antivascular endothelial growth factor therapy versus photodynamic therapy for idiopathic choroidal neovascularization. Am J Ophthalmol 155:713-9-719.e1

43. Vaz-Pereira S, Collaco L, De Salvo G, van Zeller $P$ (2015) Intravitreal aflibercept for choroidal neovascularisation in angioid streaks. Eye (Lond) 29:1236-1238

44. Hernandez-Martinez P, Dolz-Marco R, AlonsoPlasencia M, Abreu-Gonzalez R (2014) Aflibercept for inflammatory choroidal neovascularization with persistent fluid on intravitreal ranibizumab therapy. Graefes Arch Clin Exp Ophthalmol 252:1337-1339

45. Toussaint BW, Kitchens JW, Marcus DM et al (2017) Intravitreal aflibercept injection for choroidal neovascularization due to presumed ocular histoplasmosis syndrome :the HANDLE study. Retina. https://doi.org/10.1097/IAE.0000000000001590

46. Blinder KJ, Blumenkranz MS, Bressler NM et al (2003) Verteporfin therapy of subfoveal choroidal neovascularization in pathologic myopia: 2-year results of a randomized clinical trial-VIP report no. 3. Ophthalmology 110:667-673

47. Rogers AH, Duker JS, Nichols N, Baker BJ (2003) Photodynamic therapy of idiopathic and inflammatory choroidal neovascularization in young adults. Ophthalmology 110:1315-1320

48. Wachtlin J, Heimann $H$, Behme $T$, Foerster MH (2003) Long-term results after photodynamic therapy with verteporfin for choroidal neovascularizations secondary to inflammatory chorioretinal diseases. Graefes Arch Clin Exp Ophthalmol 241:899-906

49. Harissi-Dagher M, Sebag M, Gauthier D, Marcil G, Labelle P, Arbour JD (2005) Photodynamic therapy in young patients with choroidal neovascularization following traumatic choroidal rupture. Am J Ophthalmol 139:726-728

50. Chan WM, Lam DS, Wong TH et al (2003) Photodynamic therapy with verteporfin for subfoveal idiopathic choroidal neovascularization: 
one-year results from a prospective case series. Ophthalmology 110:2395-2402

51. Lipski A, Bornfeld N, Jurklies B (2008) Photodynamic therapy with verteporfin in paediatric and young adult patients: long-term treatment results of choroidal neovascularisations. Br J Ophthalmol 92:655-660

52. Peiretti E, Caminiti G, Serra R, Querques L, Pertile R, Querques G (2017) Anti-vegf therapy versus photodynamic therapy in the treatment of choroidal neovascularization secondary to central serous chorioretinopathy. Retina. https://doi.org/ 10.1097/IAE.0000000000001750

53. Chan WM, Lam DS, Lai TY et al (2003) Treatment of choroidal neovascularization in central serous chorioretinopathy by photodynamic therapy with verteporfin. Am JOphthalmol 136:836-845

54. Smretschnig E, Hagen S, Glittenberg C et al (2016) Intravitreal anti-vascular endothelial growth factor combined with half-fluence photodynamic therapy for choroidal neovascularization in chronic central serous chorioretinopathy. Eye (Lond) 30:805-811 